\title{
Overeruption in unopposed posterior teeth
}

\author{
A study of the incidence of overeruption and occlusal interferences in unopposed posterior teeth \\ H. L. Craddock and C. C. Youngson Br Dent J 2004; 196: 341-348
}

\section{Objectives}

To determine the incidence and extent of overeruption in unopposed posterior teeth; to determine the incidence of occlusal interferences associated with unopposed posterior teeth; and to detect an association between the extent of overeruption and the presence of occlusal interference.

Design and setting

The study was a single centre clinical retrospective analysis. The setting was in the Combined Care Clinic of Leeds Dental Institute. The patients were undergoing routine restorative care by final year undergraduate dental students during 2000.

Subjects and methods

All patients over18 years of age with one or more unopposed posterior tooth, were invited to participate. A sample group of 120 subjects were recruited. A clinical occlusal examination was carried out and the presence of initial RCP contacts and excursive interferences were recorded. Study models were made and digitally scanned.

Outcome measures

Measurements of overeruption from the curve of Spee were made from the images obtained, by a single observer. Reliability of the measurements was assessed by means of a Bland Altman Plot. The presence and site of retruded contact position (RCP) contacts and excursive interferences were recorded by a single observer. Reliability of the measurements was assessed by means of Kappa scores. The association between overeruption and the presence of occlusal interference was examined using Spearmans Correlation Coefficient. Results

A total of 120 subjects were recruited, with a total of 155 unopposed sites. Eighty-one of the sites belonged to male subjects, 74 to female subjects. The mean age of the group was 50.9 years, standard deviation 13.9. Eighty-three per cent of sites displayed overeruption (95\% CI 78-90), ranging from under $0.5 \mathrm{~mm}$ to $5.4 \mathrm{~mm}$. The reliability of the observations was found to be good. A total of 51.6\% (95\% CI 44-60) of unopposed teeth were involved in RCP contacts or excursive interferences. Thirty-two sites were involved in more than one contact or interference. The intra-examiner agreement was found to be good, using Kappa. The findings of the Spearman's correlation, show that if an association exists between overeruption and occlusal interference, it is weak, and of no statistical significance.

\section{Conclusions}

Eighty-three per cent of unopposed teeth are likely to overerupt, and the extent of the overeruption may be marked. The incidence and extent of overeruption is of clinical significance, not only in terms of treatment planning to prevent undesirable vertical movement, but also in the restoration of the edentulous space. The reliability of observations made in the study was found to be good, justifying the methodology. Fifty-one point six per cent of unopposed teeth are likely to be involved in RCP contacts or excursive interferences. The incidence is of clinical significance when planning the restoration of unopposed teeth, or the associated edentulous space. Only a weak statistical association was demonstrated between the degree of overeruption and the presence of occlusal interferences.

\section{COMMENT}

This paper investigated the incidence of overeruption and occlusal interferences in unopposed posterior teeth. The study was involved in an area of interest to all dental practitioners involved in the restorative management of patients as it is unclear from the present literature what the likelihood of over-eruption is, following tooth loss. The method, results and statistical analysis were appropriate and the study demonstrated that $83 \%$ of unopposed teeth are likely to over-erupt and $51.6 \%$ of unopposed teeth are likely to be involved in RCP contacts or excursive interferences. It would have been interesting if the study had also investigated the incidence of interferences in patients without missing teeth.

The authors consider that these findings are of clinical significance when planning the restoration of unopposed teeth as the removal of such contacts during tooth preparation may affect the maxillo-mandibular relationship and result in an undesirable occlusal relationship. This paper therefore has established that over-eruption of teeth is a clinical finding that should be considered when the restoration of a missing tooth is planned. The authors do not comment on whether their findings indicate that following tooth loss, the space should be restored to avoid the possibility of interferences, which is an area that might benefit from further investigation.

Dr Nick Grey, Consultant in Restorative Dentistry Edinburgh Postgraduate Dental Institute doi:10.1038/sj.bdj.4811080

\section{IN BRIEF}

- Changes in patterns of tooth loss, and an ageing population, may mean that there will be an increase in the number of partially dentate patients.

- The type and extent of occlusal change may compromise restoration of the unopposed tooth and teeth bounding the extraction site.

- Strategies to prevent undesirable tooth movements may be indicated. 